\section{Cureus}

\title{
Gram-Negative Pyomyositis in an Immunocompetent Patient
}

\author{
Michelle Knees $^{1}$, Muhammad Talha Ayub ${ }^{2}$, Ajaydas Manikkan ${ }^{2}$ \\ 1. Medical Student, Chicago College of Osteopathic Medicine 2. Internal Medicine, John H. Stroger, Jr. \\ Hospital of Cook County, Chicago, USA
}

$\square$ Corresponding author: Michelle Knees, mknees45@midwestern.edu Disclosures can be found in Additional Information at the end of the article

\section{Abstract}

Pyomyositis is an acute or sub-acute primary infection of the striated muscles. It is commonly misdiagnosed in its early stages due to its nonspecific presentation and lower suspicion among physicians when it comes to diagnosis. It has been historically associated with tropical climates but is being seen with increasing incidence in temperate regions. In both tropical and temperate areas, Staphylococcus aureus is the most common causative organism; gramnegative organisms are rare and traditionally only seen in immunocompromised patients. We report a case of Klebsiella pneumoniae pyomyositis in an immunocompetent patient with no risk factors. The awareness of the possibility of gram-negative pyomyositis in immunocompetent patients will broaden initial empiric antibiotic treatment, especially in those patients not responding to traditional empiric treatment.

Categories: Internal Medicine, Radiology, Infectious Disease

Keywords: pyomyositis, gastrocnemius, gram-negative bacteria, klebsiella pneumoniae, immunocompetent

\section{Introduction}

Pyomyositis is a primary infection of striated muscle. Most cases are reported in immunocompromised patients in tropical areas [1]. Pyomyositis in immunocompetent patients from temperate climates is a documented but rare entity [2]. It presents with non-specific symptoms-commonly fever and muscle pain. If not diagnosed, or if misdiagnosed, pyomyositis has a high morbidity and mortality rate [3]. Empiric treatment in immunocompetent patients is directed against Staphylococcus aureus and streptococci [4]. Gram-negative microbial infections have also been documented, although they are rare [5]. Given the potential for fatal sequelae following undertreated pyomyositis, clinicians should have a lower threshold for starting broader empiric treatment in immunocompetent patients.

Received 03/30/2018

Review began 03/30/2018 Review ended 03/31/2018 Published 04/09/2018

\section{(c) Copyright 2018}

Knees et al. This is an open access article distributed under the terms of the Creative Commons Attribution License CC-BY 3.0., which permits unrestricted use, distribution, and reproduction in any medium, provided the original author and source are credited.

\section{Case Presentation}

A 59-year-old female presented with a two-week history of progressively worsening right calf pain and swelling that was aggravated with movement and associated with fevers and chills. She denied any prior trauma, insect bites, and knee or ankle pain. She had taken a 12-hour international flight a week before the swelling began.

The patient's past medical history was only significant for a mitral valve prolapse; she denied any history of hypertension, diabetes, thromboembolic events, or autoimmune disorders. She endorsed smoking five cigarettes per day for the past four years but denied any use of alcohol or illicit drugs. 


\section{Cureus}

On admission, she was febrile at $101.3^{\circ} \mathrm{F}$ with otherwise normal vitals. Her physical exam was unremarkable except for a warm, erythematous, swollen, tender right calf with an overlying maculopapular rash.

Laboratory results were notable for leukocytosis, elevated erythrocyte sedimentation rate (ESR) and C-reactive protein (CRP), and normocytic anaemia. Urinalysis was normal. A lower extremity ultrasound ruled out deep venous thrombosis. Given the high suspicion for deep vein thrombosis (DVT), lower extremity computed tomography (CT) angiography was done, which revealed a lobulated collection measuring $3.1 \mathrm{~cm} \mathrm{X} 3.8 \mathrm{~cm} \mathrm{X} 10.2 \mathrm{~cm}$ within the medial gastrocnemius muscle (Figure 1). Ultrasound-guided drainage yielded $20 \mathrm{ml}$ of purulent fluid, thereby confirming the diagnosis of pyomyositis.

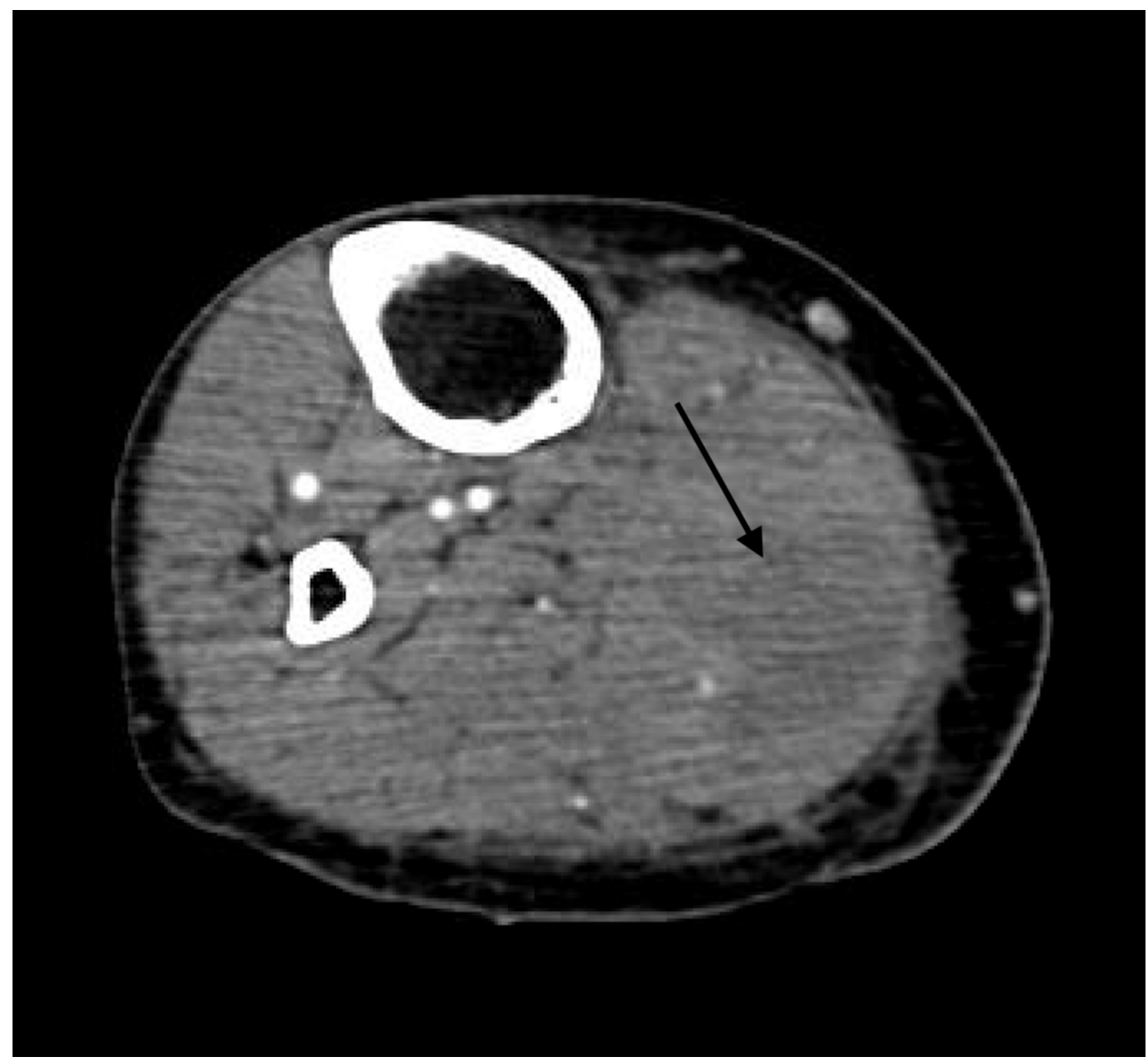

FIGURE 1: CT angiogram showing fluid collection in gastrocnemius

CT: computed tomography

One dose of empiric cefazolin and vancomycin was given in the emergency department after blood cultures were drawn. The extracted fluid was sent for culture, and the patient was treated with ceftriaxone following a second set of blood cultures.

A transthoracic echocardiogram was done next to rule out infective endocarditis as the source; 
the image results could not definitively rule out vegetations. Transesophageal echocardiography confirmed that there were no vegetations. Human immuno virus (HIV) serology was also negative.

Five days into admission, the wound cultures grew Klebsiella pneumoniae; both blood cultures remained free of growth. The patient made remarkable clinical improvement. She became afebrile, the leukocytosis resolved, and she was discharged with oral trimethoprimsulfamethoxazole. She was lost to follow-up.

\section{Discussion}

Pyomyositis is a rare bacterial infection, often with associated abscess formation in the striated muscles [1]. While common in tropical climates, it is much rarer in the United States; under 700 cases of pyomyositis have been reported in the literature [6-7]. Pyomyositis is believed to be a primary infection of skeletal muscle occurring after a transient bacteraemia without any contiguous foci from the adjacent skin, soft tissue, or bone [5, 8-9]. Interestingly, it does not usually occur in association with endocarditis or other metastatic infections [9]. Striated muscles are generally resistant to infection and the exact pathogenesis of pyomyositis is still not fully understood $[5,9]$.

Pyomyositis has classically been a disease process of tropical areas but is gaining recognition as a temperate-climate disease [1-2]. However, pyomyositis in temperate climates is often associated with an immunocompromising condition, most notably HIV. Among those who are HIV negative, nearly half have an immunocompromising condition such as diabetes mellitus, malignancy, or a rheumatological disorder [1]. Pyomyositis has also been reported after trauma, intravenous (IV) drug use, and malnutrition [2].

Staphylococcus aureus is the most common causative microbe, followed by group A streptococci; S. aureus accounts for up to $90 \%$ of tropical and $75 \%$ of temperate pyomyositis [6, 9]. Gram-negative bacilli are a much rarer cause of pyomyositis; 22 cases have been reported from 1966 through 2000, and five of those were caused by Klebsiella. Unlike our patient, the majority of these patients were immunocompromised or had known risk factors [5].

Pyomyositis traditionally progresses through three stages. Stage one is defined by crampy local muscle pain and a low-grade fever; at this stage, it is treatable with antibiotics alone [9]. However, over $90 \%$ of patients will present in stage two with extreme muscle tenderness and a frank abscess. Antibiotics and drainage are typically required at this stage. If untreated or undertreated, patients will progress to stage three, which is defined by extreme muscle pain, fluctuance, and shock $[1,4,9]$. Complications of pyomyositis include septic shock, endocarditis, pneumonia, pericarditis, septic arthritis, brain abscesses, rhabdomyolysis, and death [3, 9]. Given the high morbidity and mortality if untreated, empiric antibiotic treatment should be initiated once the clinician suspects pyomyositis $[2,4,10]$. Traditionally, immunocompetent patients have been treated empirically with broad spectrum antibiotics which cover $\mathrm{S}$. aureus and streptococci until culture-directed therapy can be initiated [4, 10]. However, this case emphasizes that clinicians should have a low threshold for initiating broader empiric treatment to cover for gram-negative species, even in immunocompetent patients.

\section{Conclusions}

Pyomyositis has historically been associated with tropical climates but is now being seen with increasing frequency in temperate areas. When found in an immunocompetent patient, empiric antibiotics which target $\mathrm{S}$. aureus and streptococci are usually initiated; immunocompromised patients traditionally receive broader-spectrum antibiotics which additionally cover gramnegative bacteria. Given the devastating sequelae of undertreated pyomyositis, physicians 
should consider starting or switching to antibiotics which cover gram-negative bacteria in any immunocompetent patient who is not improving under traditional antibiotics.

\section{Additional Information \\ Disclosures}

Human subjects: Consent was obtained by all participants in this study. Conflicts of interest: In compliance with the ICMJE uniform disclosure form, all authors declare the following:

Payment/services info: All authors have declared that no financial support was received from any organization for the submitted work. Financial relationships: All authors have declared that they have no financial relationships at present or within the previous three years with any organizations that might have an interest in the submitted work. Other relationships: All authors have declared that there are no other relationships or activities that could appear to have influenced the submitted work.

\section{References}

1. Crum NF: Bacterial pyomyositis in the United States. Am J Med. 2004, 117:420-428. 10.1016/j.amjmed.2004.03.031

2. Comegna L, Guidone PI, Prezioso G, et al.: Pyomyositis is not only a tropical pathology: a case series. J Med Case Rep. 2016, 10:372. 10.1186/s13256-016-1158-2

3. Sharma A, Kumar S, Wanchu A, et al.: Clinical characteristics and predictors of mortality in 67 patients with primary pyomyositis: a study from North India. Clin Rheumatol. 2010, 29:45-51. 10.1007/s10067-009-1277-x

4. Agarwal N, Aroor S, Saini P, Gupta A, Kaur N: Pyomyositis: are we missing the diagnosis? Surg Infect (Larchmt). 2016, 17:615-621. 10.1089/sur.2015.191

5. Wang T, Wong S, Woo P: Two cases of pyomyositis caused by Klebsiella pneumoniae and review of the literature. Eur J Clin Microbiol Infect Dis. 2001, 20:576-580.

$10.1007 /$ s100960100556

6. Small LN, Ross JJ: Tropical and temperate pyomyositis. Infect Dis Clin North Am. 2005, 19:981-989. 10.1016/j.idc.2005.08.003

7. Bickels J, Ben-Sira L, Kessler A, Wientroub S: Primary pyomyositis. J Bone Joint Surg Am. 2002, 84:2277-2286.

8. Christin L, Sarosi GA: Pyomyositis in North America: case reports and review. Clin Infect Dis. 1992, 15:668. 10.1093/clind/15.4.668

9. Crum-Cianflone NF : Bacterial fungal, parasitic, and viral myositis. Clin Microbiol Rev. 2008, 21:473-494. 10.1128/CMR.00001-08

10. Liao WH, Lai CC, Huang SH, Lin SH: Rectus femoris pyomyositis caused by Klebsiella pneumoniae. Surg Infect. 2014, 15:464-465. 10.1089/sur.2013.183 